\title{
Nouvelles Espèces, nouvelles localisations de Culicoides (Dipt. Ceratop.) du Midi de la France
}

\author{
Par J. CALlot, M. KREMER, B. MOLET et A. BACH \\ (avec la collaboration technique de A. VernedE) \\ (Institut de Parasitologie de la Faculté de Médecine de Strasbourg. Directeur: Pr J. Callot)
}

\section{Résumé}

Nous rapportons la présence ou donnons de nouvelles localisations dans le midi de la France des espèces suivantes:

C. pumilus, C. derisor, C. heliophilus, C. haranti, C. begueti, C. cataneii, C. odibilis, C. clastrieri, C. gejgelensis, C. musilator, C. obsoletus, C. fagineus, C. pulicaris (?), C. subfascipennis, C. heteroclitus, $C$. cubitalis, $C$. sylvarum, $C$. semimaculatus, $C$. longipennis, C. circumscriptus.

C. kurekstaicus, C. tauricus, C. gejgelensis sont signalés pour la première fois en France.

C. dzhafarovi est une (nouvelle) espèce dédiée à Dzhafarov qui l'avait décrite sans la séparer nettement de $C$. heliophilus.

Nous décrivons $C$. virdoulensis $\mathrm{n}$. sp. proche de $C$. cubitalis et de $C$. saevanicus.

A propos de C. cubitalis, nous pensons avoir affaire à un complexe d'espèces, dont nous indiquons les limites sans pouvoir les séparer. Nous indiquons des particularités morphologiques variables de $C$. longipennis et $C$. pulicaris.

\section{Summary}

New localisations of the following species were found in the South of France.

C. pumilus, C. derisor, C. heliophilus, C. haranti, C. begueti, C. cataneii, C. odibilis, C. clastrieri, C. gejgelensis, C. musilator, C. obsoletus, C. fagineus, C. pulicaris (?), C. subfascipennis, C. heteroclitus, C. cubitalis, C. sylvarum, C. semimaculatus, C. longipennis, C. circumscriptus.

C. kurckstaicus, C. tauricus, C. gejgelensis are reported for the first time in France.

C. dzhafarovi is a new species dedicated to Dzhafarov who gave a description of it, but did not separate it definitely from C. heliophilus.

C. vidourlensis $\mathrm{n} . \mathrm{sp}$. is described ; it' appears very near from C. cubitalis and C. saevanicus.

C. cubitalis is probably a complex of species the limits of which are indicated by the authors.

Morphological particularities of $C$. longipennis and $C$. pulicaris are reported. 
La faune des Culicoides de la région méditerranéenne réserve de nombreuses surprises et pose des problèmes plus nombreux encore. Les exemplaires étudiés dans cette note ont été obtenus de la façon habituelle, soit par éclosion à partir de boues et sables de gîtes larvaires, soit par des captures à la lumière ou exceptionnellement sur appât.

\section{Culicoides pumilus Winn. sensu Callot et Kremer.}

Localité :

- Une femelle sur appât humain, le Rayol (Var), juin 1965.

- Mâles et femelles, bord du Vidourle, en aval de Sommières (Gard) (par éclosion), avril 1966.

- Un mâle, par éclosion, bord de ruisseau, N. 555 à $2 \mathrm{~km}$ au Nord de Montferrat (Var).

\section{Culicoides derisor Callot et Kremer.}

Localité :

- Une femelle, prise au piège lumineux, Le Rayol (Var), juin 1965.

\section{Culicoides beliopbilus EDw.}

\section{Localite :}

- Une femelle éclose de boues prélevées près d'une source, non abritée, Mont Aigoual, $1.500 \mathrm{~m}$, mai 1966 .

\section{Culicoides kurekstaicus Dzhafarov.}

Cette espèce est signalée en France pour la première fois, elle est connue d'Azerbaïdjan et de Tunisie (Callot, Kremer, Rioux et Juminer).

Les dessins du thorax sont plus importants, surtout chez l'un des deux exemplaires, que dans la description originale et que chez nos exemplaires de Tunisie.

\section{Localité :}

- Eclosion de deux mâles à partir de boues d'une mare résiduelle du lit du Verdon près du pont d'Aiguine, Basses-Alpes, $465 \mathrm{~m}$, mai 1966.

\section{Culicoides tauricus Gutzevich.}

Nous n'en connaissons que les femelles écloses de divers gîtes. Les spermathèques sont de formes particulières et semblables aux schémas qu'en donne Dzhafarov, elles ne sont pas chitinisées. Signalons que les femelles de $C$. engubandei, sont également très proches de nos exemplaires. 
Cependant, des arguments biogéographiques et la morphologie du thorax, ici dépourvu de taches, nous amènent à déterminer nos exemplaires comme $C$. tauricus.

\section{Localité :}

- Une femelle éclose de boues du lit de l'Artuby (N. 555) à 758 m (Basses-Alpes).

- Une femelle éclose de boues du Verdon, Pont de Soleil à $652 \mathrm{~m}$ (Basses-Alpes).

- Une femelle éclose d'un fossé d'irrigation à Bras-d'Asse (Basses-Alpes).

\section{Culicoides dzhafarovi $\quad$ n. sp.}

Nous dédions cette espèce nouvelle à Dzhafarov qui l'a déjà décrite en l'assimilant à $C$. heliophilus Edw.

Toutes les descriptions de son $C$. aff. heliophilus sont parfaitement celles de notre $C$. dzhafarovi et nous nous bornerons à indiquer les différences entre cette espèce et C. heliophilus sensu stricto.

MâLE.

L'ædeagus de $C$. dzhafarovi présente un corps beaucoup plus long, trapézoïdal, que celui de $C$. heliophilus, il est également bien plus large que celui de C. pseudoheliophilus.

L'extrémité des paramères se termine plus rapidement que celle de $C$. heliophilus et la base est différente de celle de $C$. pseudoheliophilus.

\section{Femelle.}

Elle est intermédiaire entre celle de $C$. heliophilus et celle de $C$. pseudoheliophilus.

La disposition des sensilles des antennes pour ces trois espèces et pour $C$. inornatipennis est la suivante :

\begin{tabular}{|c|c|c|c|c|c|c|c|c|c|c|c|c|c|}
\hline $\begin{array}{l}\text { Artcles } \\
\text { antennaires .. }\end{array}$ & 3 & 4 & 5 & 6 & 7 & 8 & 9 & 10 & 11 & 12 & 13 & 14 & 15 \\
\hline C. heliophilus & 2,3 & 0 & 0 & 0 & 0 & 0 & 0 & 0 & 1 & 1 & 1 & 1,9 & 1,44 \\
\hline $\begin{array}{l}\text { C. pseudo- } \\
\text { heliophilus .. }\end{array}$ & 1,5 & 0 & 0 & 0 & 0 & 0 & 0 & 0 & 0,9 & 0,02 & 1 & 1,1 & 1,4 \\
\hline C. dzhafarovi. & 3 & 0 & 0 & 0 & 0 & 0 & 1 & 0 & 1 & 1 & 1 & 1 & 0 \\
\hline $\begin{array}{l}\text { C. inornati- } \\
\text { pennis ...... }\end{array}$ & 6 & 0,5 & 1 & 1,2 & 1 & 1 & 2 & 1,3 & 2,2 & 3,2 & 3,1 & 4 & 3 \\
\hline
\end{tabular}

L'indice antennaire de $C$. dzhafarovi est compris entre 1,06 et 1,27. La longueur totale de l'antenne varie entre $390 \mu$ et $440 \mu$. Ces dimensions sont inférieures à celles trouvées par Dzhafarov. 


\section{Localité :}

- Très nombreux mâles et femelles, éclos de boues de ruisseau prélevées sous un pont de la D. 48 (entre Vidauban et la Garde, Var), mai 1966. 1966.

- Une femelle éclose d'un bord de ruisseau, à La Palud (Basses-Alpes), mai

C. baranti Rioux, Descous et $\mathrm{PECH}$.

Localité :

- Une femelle capturée au piège lumineux, Le Rayol (Var), juin 1965.

\section{C. begueti Clastrier.}

\section{Localité :}

- Mâles et femelles éclos d'une mare résiduelle d'un ruisseau, dans du grès, à 15 km de la Garde Freinet (Maures, Var), mai 1966.

\section{C. cataneii CLASTRIER.}

Bien que la forme du palpe, la disposition des taches alaires et les indices diffèrent chez les femelles de $C$. begueti, $C$. haranti et $C$. cataneii, nous ne pouvons donner ces diagnoses avec certitude, la disposition des sensilles étant très proche chez ces trois espèces, et les éléments morphologiques précédents assez variables.

\section{Localité :}

- Une femelle éclose d'un canal d'irrigation sur la D. 173 à $19 \mathrm{~km}$ à l'Est de Cavaillon, mai 1966. (Vaucluse).

- Une femelle (?) éclose du même gîte que $C$. begueti, mai 1966.

\section{Culicoides odibilis AustıN.}

Les dessins du thorax sont souvent régressés.

\section{Localité :}

- Mâles et femelles éclos du bord de l'Encrème à $1 \mathrm{~km}$ de Céreste (Vaucluse).

- Mâles et femelles éclos du gîte de la D. 48 (Massif des Maures).

- Un mâle éclos du gîte de C. begueti, mai 1966.

\section{Culicoides clastrieri Callot, Kremer et Déduit).}

Localité :

- Une femelle éclose du bord de l'Encrème, Céreste (Vaucluse), mai 1966. 


\section{Culicoides gejgelensis Dzhafarov.}

Cette espèce est diagnostiquée avec les mêmes restrictions que pour sa détermination en Italie.

Les taches des ailes et la forme de l'aedeagus sont un peu différentes de la description originale, sans permettre toutefois d'en faire une espèce nouvelle.

\section{Localité :}

- Mâles et femelles éclos du gîte de la D. 48 (Var), mai 1966.

- Mâles et femelles du gîte de $C$. begueti (Maures).

- Mâles et femelles éclos d'un bord de ruisseau sur terrain riche en bauxite, sur le Mont de la Loube (Var), mai 1966.

\section{Culicoides musilator Kremer et CAllot.}

Localité :

- Femelles écloses de boues du Vidourle, à $300 \mathrm{~m}$ en aval de Sommières (Gard), avril 1966.

-- Mâles et femelles, bord de l'Encrème, Céreste (Vaucluse), mai 1966.

- Mâle, bord de ruisseau, Lourmarin (Vaucluse), mai 1966.

- Mâles, gîte de la D. 48 (cité plus haut), mai et septembre 1966.

\section{Culicoides obsoletus MEIGEN}

Localité :

- Un mâle capturé au piège lumineux à la Garde Freinet (Massif des Maures, Var), août 1966.

\section{Culicoides fagineus EDWARDs.}

Localité :

- Une femelle capturée au piège lumineux, Le Rayol, juin 1966.

\section{Culicoides pulcaris (L.)?}

Un mâle et de nombreuses femelles présentent des caractères intermédiaires entre ceux de $C$. halophilus et $C$. pulicaris. La base de la cellule M1 est fortement tachée, parfois entièrement brune comme chez $C$. halophilus. Les longueurs des ailes sont également celles de cette espèce. La forme de la tache en diabolo de r5 est variable, assez proche en général, de celle de $C$. pulicaris et les petites taches sombres à l'extrémité des nervures M1 et M2 ne sont pas marquées d'ocelles. Les thorax n'ont pas pu être examinés. 


\section{Localité :}

- Piège lumineux, Le Rayol, Var, Juin 1965.

\section{Culicoides subfascipennis analis KIEFFER.}

Localité :

- Une femelle capturée au piège lumineux, Le Rayol, juin 1965.

- Une femelle éclose de boue d'une mare résiduelle dans le lit d'un ruisseau à sec, sur grès, à $15 \mathrm{~km}$ de la Garde-Freinet (Massif des Maures, Var).

\section{C. beteroclitus Callot et Kremer.}

Localités :

- Mâles et femelles éclos de sables du Bourrian, ruisseau près de St-Tropez (Var); juin 1966.

- Mâles et femelles de gîte de la route D. 48 mai et septembre 1966 ; de boue d'un ruisseau, près de la D. 74, mai 1966 (Massif des Maures, Var).

\section{Culicoides cubitalis EDwards.}

Nous nous trouvons à propos de cette espèce, en face d'un complexe comprenant plusieurs variantes mâles et plusieurs variantes femelles. L'aedeagus peut prendre la forme mince, allongée, sans membrane entre les bras, ou au contraire, la forme large, avec membrane importante entre les bras (cf. variété de C. cubitalis in Kremer, 1965). La membrane basale habituellement non spiculée peut présenter un ou plusieurs spicules.

Les femelles ont des indices antennaires qui varient entre 1,17 et 1,76, avec une répartition régulière en courbe de Gauss dont la moyenne serait entre 1,45 et 1,55 . De plus, alors que $C$. cubitalis est décrit comme n'ayant pas de macrotriches dans la cellule basale de l'aile, certains de nos exemplaires en présentent quelques-unes.

Il ne nous est pas possible de schématiser ces variations, aussi donnons-nous les localisations sans préciser le type morphologique.

\section{Localité :}

- Mâles et femelles, boues du Vidourle, en aval des Sommières (Gard), avril 1965 ; mare dans le lit de la Nartuby (N. 555) au niveau de Chateaudouble ; bord de ruisseau sur la route de N.-D.-des Anges ; source près d'une mine de bauxite, Mont de la Loube ; mare résiduelle d'un ruisseau, sur grès, à $15 \mathrm{~km}$ de la Garde-Freinet ; gîte de la D. 48 ; lit de l'Artuby, route N. 555 (Var); bord de ruisseau à eau ferrugineuse entre Alès et Générargues (Gard) ; bord de ruisseau sous-bois, près de Lourmarin (Vaucluse) ; bord de ruisseau à Lourmarin, bord de mare à Lourmarin. (Tous ces prélèvements en mai 1966). 


\section{Culicoides sylvarum Callot et Kremer.}

Localité :

- Mâles et femelles capturés au piège lumineux, Le Rayol (Var).

\section{Culicoides semimaculatus CLASTRIER.}

Localité :

— Mâles et femelles capturés au piège lumineux, Le Rayol, Var, juin 1965.

\section{Culicoides longipennis KHALAF.}

Si la diagnose des mâles de cette espèce pose peu de problèmes, celle des femelles est plus compliquée que nous ne l'avions supposé. En effet, la disposition des sensilles semble très variable chez cette espèce, il peut $\mathrm{y}$ avoir des sensilles sur tous les articles de 3 à 10 , mais ils peuvent, contrairement à ce que nous avons écrit, comme chez C. similis, manquer sur les articles 4 et 6 .

\section{Localité :}

- Mâles et femelles, de boues prélevées dans le Bourrian, près de St-Tropez, juin 1965 ; de boues du Vidourle, en aval de Sommières (Gard) prélevées en décembre 1964 ; gîte de la route D. 48 (Maures) en mai et septembre 1966; dans une mare du lit de la Nartuby, près de Chateaudouble (Var); dans une mare résiduelle du lit du Verdon, près du pont d'Aiguine (Basses-Alpes) ; bord de l'Encrème, près de Céreste ; bord de mare à Lourmarin (Vaucluse).

Ces derniers prélèvements faits en mai 1966.

\section{Culicoides circumscriptus KIEFFER.}

Localité :

- Un mâle éclos du lit de la Garde, D. 14 (Var), juin 1965 ; bord de ruisseau à La Palud (Basses-Alpes), mai 1966 ; gîte de la D. 48, mai 1966 (cf. supra).

\section{Culicoides vidourlensis n. $\mathrm{sp}$.}

Dans les boues du Vidourle (Sommières, Gard), nous avons trouvé une espèce de Culicoides, s'apparentant par ses ailes au groupe carjalaensis, par son hypopygium à C. cubitalis et originale par la disposition des sensilles des femelles.

Caractères communs aux deux sexes:

Thorax dépourvu de dessins notables.

Ailes grises présentant deux rangées antéro-postérieures de tâches claires réparties de la façon suivante : 
Première rangée : une tache sur le tiers proximal du bord antérieur et entamant la première cellule radiale, plus ou moins continue avec une tache claire située entre $M$ et $\mathrm{Cu}$, une tache à l'extrémité de la cellule ánale.

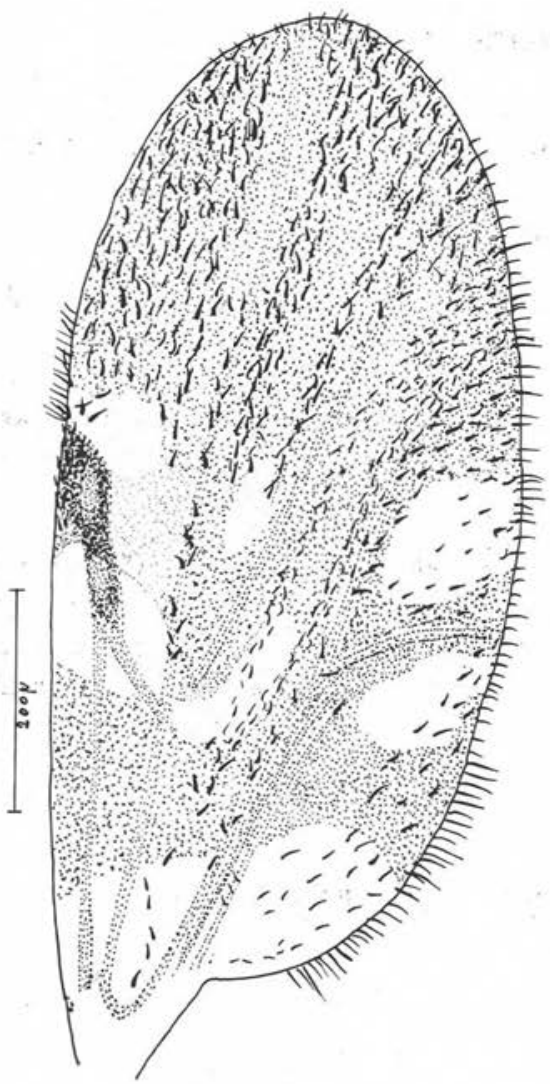

F1G. 1. - Culicoides vidourlensis: aile de la femelle

Deuxième rangée: une tache juste après la deuxième cellule radiale, une tache dans la base de la cellule $\mathrm{ml}$ (fourche médiane), une tache dans $\mathrm{Cu}$. Une tache également à la base de l'aile.

Les ailes sont pourvues de macrotriches qui sont cependant absentes des cellules sous-costales, basales et à la base de l'aile.

Peigne tibial à quatre soies principales. 
MâLE.

Taches alaires plus larges et moins nettement délimitées, macrotriches moins nombreuses.

Longueur totale de l'aile : $1 \mathrm{~mm}$; costa : 0,55 mm ; largeur : 0,39 mm.

Hypopygium : Aspect général et tous les détails semblables à ceux de l'hypopygium de $C$. cubitalis.

Lamelle entaillée, processus digitiformes divergents, sternite profondément entaillé en pente douce.

Coxites de forme habituelle à apodème dorsal important et apodème ventral long, fin et droit (Celui de $C$. cubitalis est peut-être plus sinueux); aedeagus à bras bien chitinisés, corps long à bords parallèles.

Paramères débutants par une base importante bien chitinisée en équerre, puis une partie médiane modérément renflée et finalement de plus en plus fins et contournés.

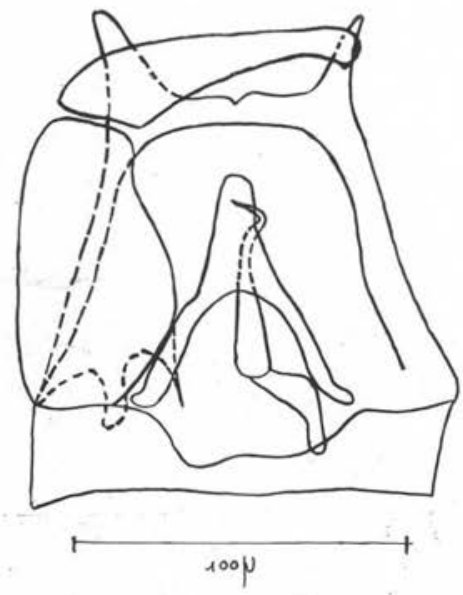

Fig. 2. - Culicoides vidourlensis: hypopygium mâle

Membrane basale nue. Style de forme habituelle. Longueur $89 \mu$. Pattes (mensurations en microns).

\begin{tabular}{|c|c|c|c|c|c|c|c|}
\hline & F & $\mathrm{T}$ & 1 & 2 & 3 & 4 & 5 \\
\hline Ant. & 319 & 333 & 166 & 99 & 66 & 46 & 44 \\
\hline Moy. & 413 & 420 & 229 & 96 & 61 & 44 & 48 \\
\hline Post. & 420 & 435 & 239 & 103 & 68 & 44 & 48 \\
\hline
\end{tabular}

Peigne tibial, longueur des soies principales : 48, 35, 33, $24 \mu$. 


\section{Femelle.}

Taches alaires moins étendues, macrotriches plus abondantes.

Longueur totale de l'aile : $1 \mathrm{~mm}$; costa : 0,57 mm ; largeur : 0,47 mm.

Tête: yeux glabres, espace interoculaire large (plus large qu'une facette).

Troisième article du palpe assez bien renflé, à fossette sensorielle unique, limitée par une petite bordure.

Longueur des articles:

\begin{tabular}{|r|r|r|r|}
\hline $1+2$ & 3 & 4 & 5 \\
\cline { 1 - 3 } & 59 & 22 & 28 \\
\hline
\end{tabular}

Longueur totale : $157 \mu$.

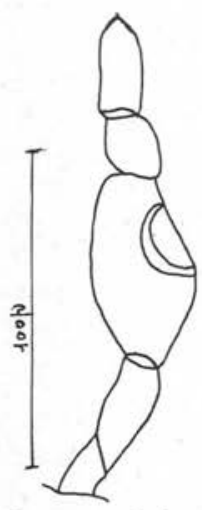

FIG. 3. - Culicoides vidourlensis: palpe de la femelle

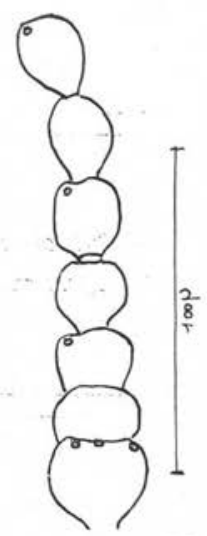

Fig. 4. - Culicoides vidourlensis: premiers articles antennaires de la femelle

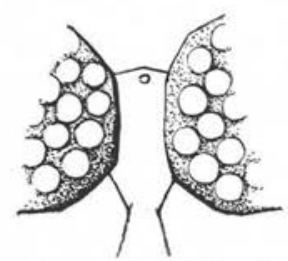

Fig. 5. - Culicoides vidourlensis: espace interoculaire de la femelle

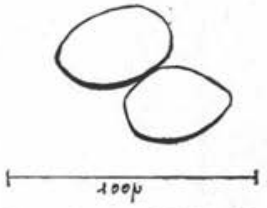

FIG. 6. - Culicoides vidourlensis : spermathèques

\section{Antennes:}

Répartition des sensilles et longueur des articles.

\begin{tabular}{|l|c|c|c|c|c|c|c|c|c|c|c|c|c|c|}
\hline $\begin{array}{l}\text { Articles } \\
\text { antennaires }\end{array}$ & 3 & 4 & 5 & 6 & 7 & 8 & 9 & 10 & 11 & 12 & 13 & 14 & 15 \\
\hline \begin{tabular}{ll} 
Sensilles \\
\hline Longueur
\end{tabular} & $\frac{3,8}{29,6}$ & $\frac{0}{22,2}$ & $\frac{1}{20,3}$ & $\frac{0,2}{22,2}$ & $\frac{1}{22,2}$ & $\frac{0}{24}$ & $\frac{1}{25,9}$ & $\frac{0}{22,2}$ & $\frac{1}{51,8}$ & $\frac{1}{55,5}$ & $\frac{1}{55,5}$ & $\frac{1}{59,2}$ & $\frac{1}{85,1}$ \\
\hline
\end{tabular}


Les sensilles sont la moyenne de 8 exemplaires, les longueurs sont celles du cotype. Longueur totale de l'antenne 496 microns.

Indice antennaire: 1,62 .

\section{Pattes :}

\begin{tabular}{|c|c|c|c|c|c|c|c|}
\hline & M & $\mathrm{T}$ & 1 & 2 & 3 & 4 & 5 \\
\hline Ant. & 319 & 319 & 159 & 81 & 55 & 37 & 48 \\
\hline M. & 377 & 377 & 188 & 96 & 59 & 63 & 48 \\
\hline Post. & 377 & 384 & 188 & 107 & 61 & $48^{\circ}$ & 55 \\
\hline
\end{tabular}

Longueur des épines du peigne tibial: 48, 52, 41, 29 microns.

\section{Spermathèques :}

Deux spermathèques sensiblement égales de $48,1 \times 37 \mu$ et $47,1 \times 39 \mu$ et une spermathèque rudimentaire de 11 microns de long.

Type et cotype déposés à l'Institut de Parasitologie de la Faculté de Médecine de Strasbourg.

\section{Discussion}

La diagnose des mâles est faite sur la présence d'un hypopygium analogue à celui de $C$. cubitalis et d'une tache claire bien visible dans la cellule $\mathrm{m} 1$.

Les femelles peuvent être diagnostiquées par les caractères des ailes et la répartition particulière des sensilles antennaires. Seules, $C$. sylvarum et $C$. saevanicus ont une telle répartition parmi les espèces paléarctiques et les ailes et les indices antennaires entre autres caractères sont différents.

\section{Bibliographie}

Callot (J.) et Kremer (M.), 1965. - Sur quelques Diptères Nématocères du Var, avec la description d'espèces nouvelles de Culicoides (Ceratopogonidae). Ann. Par. Hum. Comp., 40, 329-339.

—, - 1961. - Scission de l'espèce Culicoides truncorum Edwards (Diptera: Ceratopogonidae) en deux espèces. Bull. Soc. Path. Exot., 54, 389-398.

-, - et ColuzzI (M.), 1965. Nouvelle contribution à l'étude des Culicoides (Diptères, Ceratopogonidés) d'Italie, Parassitologia, 7, 161-171. 
-, - et Dedurt (Y.), 1962. - Nouvelles espèces et nouvelles localisations de Culicoides (Diptera-Ceratopogonidae) des Ardennes, du Centre de la France, du Jura français et du Jura suisse. Ann. Par. Hum. Comp., 37, 153-171.

-, - et Rioux (J.-A.), 1963. - Sur des Culicoides (Diptera-Ceratopogonidae) dont une espèce et une variété nouvelle du Midi de la France. Ann. Par. Hum. Comp., 38, 121-129.

- , - , - et Juminer (B.), 1964. - Contribution à l'étude des Culicoides (Diptera, Ceratopogonidae) de Tunisie. Arch. Inst. Past. Tunis, 41, 357-364.

Campbell (J. A.) et Pelham-Clinton (E. C.), 1959-1960. - A taxonomic review of British species of Culicoides Latreille (Diptera, Ceratopogonidae). Proc. R. Soc. Edin., 67, 181-302.

Clastrier (J.), 1957. - Notes sur les Cératopogonidés: H. Quelques Culicoides d'Algérie à ailes tachetées. Arch. Inst. Past. Alg., 35, 404-444; 1958, III. Culicoides semimaculatus n. sp. d'Algérie. Arch. Inst. Past Alg., 36, 55-60.

Coluzzi (M.) et KREMer (M.), 1964. - Contributo alla conoscenza dei culicoidi italiani (Diptera Ceratopogonidae). Parassitologia, 6, 63-69.

Dzhafarov (S. M.), 1964. - Krovososuscia Mokrecy (Diptera: Heleidae) Zakavkazjia. Izdateljstvo Akademii Naük Azerbajdzhanskoi SSR, 441 p., Baku.

Edwards (F. W.), 1939. - Ceratopogonidae in Edwards, Smart et Oldroyd \& British bloodsucking flies », British Museum, Londres.

Gutzevich (A. V.), 1960. - Krovososucie Mokrecy (Diptera : Heleidae) Fauny SSSR, Akad. Nauk, SSSR Opred Fauny, 72, 130 p.

KhalaF (K. T.), 1957. - Heleids from Iraq with description of new species. Bull. Soc. Entomol. Egypte, 41, 335-350.

KREMER (M.), 1966. - Contribution à l'étude du genre Culicoides Latreille, particulièrement en France, Ed. P. Lechevalier, Paris. 